\title{
Comparison of two different carbon nanotube-based surfaces with respect to potassium ferricyanide electrochemistry
}

\author{
Irene Taurino $^{\mathrm{a}, \mathrm{b}, *}$, Sandro Carrara ${ }^{\mathrm{a}}$, Mauro Giorcelli ${ }^{\mathrm{b}}$, Alberto Tagliaferro ${ }^{\mathrm{b}}$, Giovanni De Micheli ${ }^{\mathrm{a}}$ \\ a Laboratory of Integrated Systems, EPFL - École Polytechnique Fédérale de Lausanne, 1015, Lausanne, Switzerland \\ b Department of Physics, Polytechnic of Turin, 10129, Torino, Italy
}

\section{A R T I C L E I N F O}

Article history:

Received 16 May 2011

Accepted 1 September 2011

Available online 10 September 2011

\section{Keywords:}

Multi-walled carbon nanotubes

Cyclic voltammetry

Potassium ferricyanide

Electrochemical characterization

\begin{abstract}
A B S T R A C T
This paper describes the electrochemical investigation of two multi-walled carbon nanotube-based electrodes using potassium ferricyanide as a benchmark redox system. Carbon nanotubes were fabricated by chemical vapor deposition on silicon wafer with camphor and ferrocene as precursors. Vertically-aligned as well as islands of horizontally-randomly-oriented carbon nanotubes were obtained by varying the growth parameters. Cyclic voltammetry was the employed method for this electrochemical study. Vertical nanotubes showed a slightly higher kinetic. Regarding the sensing parameters we found a sensitivity for vertical nanotubes almost equal to the sensitivity obtained with horizontally/randomly oriented nanotubes $\left(71.5 \pm 0.3 \mu \mathrm{A} /(\mathrm{mM} \mathrm{cm})^{2}\right)$ and $62.8 \pm 0.3 \mu \mathrm{A} /\left(\mathrm{mM} \mathrm{cm}^{2}\right)$, respectively). In addition, values of detection limit are of the same order of magnitude. Although tip contribution to electron emission has been shown to be greatly larger than the lateral contribution on single carbon nanotubes per unit area, the new findings reported in this paper demonstrate that the global effects of nanotube surface on potassium ferricyanide electrochemistry are comparable for these two types of nanostructured surfaces.
\end{abstract}

(C) 2011 Elsevier B.V. All rights reserved.

\section{Introduction}

Carbon nanotubes (CNTs) have recently attracted a lot of interest in recent years due to good chemical stability, excellent mechanical strength, and extraordinary electrical conductivity [1]. CNTs have raised a considerable interest in electrochemistry as well. The CNT ability to increase electron transfer kinetics [2], to increase the electroactive surface area [3] and to increase the sensitivity of electrochemical [4] and conductometric sensors [5] is well-known. However the electrocatalytic properties of CNTs are strongly influenced by many factors, such as the synthesis methods and procedures to prepare the CNT-modified electrodes [4,6]. For instance, CNT-based electrodes behave differently following the CNT edge-like site number and pretreatments [7]. The electron transfer on CNTs takes place at their tips [8]. The role of CNT walls is still under investigation $[9,10]$. The objective of this paper, consequently, is to add insight by an electrochemical study of two electrodes nanostructured with differently oriented treated CNTs.

To this objective, we fabricated multi-walled carbon nanotubes (MWCNTs) directly onto silicon substrates by a chemical vapor deposition (CVD) process. We obtained vertically aligned (VA) and islands of horizontally/randomly oriented (HRO) CNTs by suitable variation of the growth parameters. We investigated acid-treated CNTs using

\footnotetext{
* Corresponding author. Tel.: +41 762657 195; fax: +4121 6934225 . E-mail addresses: irene.taurino@ep.ch (I. Taurino), sandro.carrara@epfl.ch (S. Carrara), mauro.giorcelli@polito.it (M. Giorcelli), alberto.tagliaferro@polito.it (A. Tagliaferro), giovanni.demicheli@epfl.ch (G. De Micheli).
}

the contact angle technique to demonstrate the alterations the acid treatment produces on the CNT sidewalls. Finally, we evaluated the electrochemical properties of the two nanostructured CNT-based electrodes using cyclic voltammetry (CV) of potassium ferricyanide $\left(\mathrm{K}_{4} \mathrm{Fe}(\mathrm{CN})_{6}\right)$, a redox model compound.

\section{Experimental procedure}

\subsection{Synthesis of carbon nanotubes onto silicon}

To grow MWCNTs directly onto a silicon wafer (Si-mat, Germany) with $10.16 \mathrm{~cm}$ of diameter we used a catalytic CVD process. We selected commercial camphor as carbon precursor since the 3D structure of this material facilitates the formation of nanotube rings [11] and is non-toxic for humans and the environment [12,13]. We selected ferrocene ( $98 \%$ purity in weight, Aldrich) as catalyst because it creates clusters of iron atoms on which CNTs can grow. The reactor consisted of a furnace (Kenosistec, Milan, Italy) with two separate evaporation chambers where the precursors were heated. The silicon substrate was placed on a graphite plate rotating at $12 \mathrm{rms}$ in a deposition chamber (IONVACPROCESS SRL, Rome, Italy) and heated up at the desired temperature. To obtain the two different CNT structures, we proceeded as follows.

- HRO MWCNTs. The substrate was heated at $925^{\circ} \mathrm{C}$. Ferrocene and camphor were introduced in the deposition chamber and supplied for 10 min using only their evaporation pressure with no carrier 
gas. After the deposition, the substrate was kept at the growth temperature for $30 \mathrm{~min}$ and then cooled down to room temperature.

- VA MWCNTs. The substrate was kept at $850{ }^{\circ} \mathrm{C}$. Ferrocene was introduced for $3 \mathrm{~min}$ and was supported by a laminar flow of nitrogen $\left(0.83 \mathrm{~cm}^{3} / \mathrm{s}\right)$. Camphor was simultaneously introduced with the ferrocene in the deposition chamber for 10 min with no carrier gas. The substrate was cooled to room temperature inside the chamber in inert atmosphere immediately after the deposition.

\subsection{Chemicals}

We used Phosphate Buffer Saline (PBS, Sigma) at pH 7.4 to prepare all solutions. After the deposition, we treated MWCNTs in $6 \mathrm{M} \mathrm{H}_{2} \mathrm{SO}_{4}$ (Sigma, 95-98\% vol) solution for $6 \mathrm{~h}$ [14]. We utilized potassium ferricyanide in powder form from Sigma.

\subsection{FE-SEM and contact angle}

To acquire FE-SEM images, we used a SUPRA ${ }^{\mathrm{TM}} 40$ (ZEISS) having a nominal resolution of $1.5 \mathrm{~nm}$ at $10 \mathrm{kV}$. We measured contact angles using PBS drops ( $20 \mu \mathrm{l})$ cast onto the surface of MWCNT-based electrodes before and after the treatment. The images were acquired with a digital camera. For each sample, we averaged five measurements performed from five different images.

\subsection{Electrochemical measures}

To perform CV measurements we used a Versastat 3 potentiostat (Princeton Applied Technologies). We utilized a conventional electrochemical cell with a three electrode configuration. A platinum wire served as counter electrode and $\mathrm{ag} \mid \mathrm{AgCl}$ wire saturated with $\mathrm{KCl}(3 \mathrm{M})$ as reference electrode (Roschi Rohde and Schwarz AG, Switzerland). We used only chemically treated MWCNTs on silicon substrate as working electrodes. All experiments were carried out under aerobic conditions at room temperature using PBS $0.01 \mathrm{M}$ as electrolyte support solution.

Among different common benchmark redox systems, $\mathrm{K}_{4} \mathrm{Fe}(\mathrm{CN})_{6}$ was selected for its "surface sensitive" electrochemical response, particularly for carbon materials [15]. CV was performed by varying concentrations of $\mathrm{K}_{4} \mathrm{Fe}(\mathrm{CN})_{6}$. A potential of $+300 \mathrm{mV}$ causes the oxidation of this compound according to the reaction

$\left[\mathrm{Fe}(\mathrm{CN})_{6}\right]^{-3}+e^{-} \rightarrow\left[\mathrm{Fe}(\mathrm{CN})_{6}\right]^{-4}$.

For a reversible reaction at $25^{\circ} \mathrm{C}$ the peak current, $I_{p}$, can be computed referring to the Randles-Sevcik equation [16]

$I_{p}=2.69 \cdot 10^{2} A D^{1 / 2} n^{3 / 2} v^{1 / 2} C$

where $I_{p}$ is the peak current, $A$ is the electroactive area, $D$ is the diffusion coefficient of the analyte, $n$ is the number of electrons involved in the redox reaction, $v$ is the scan rate, and $C$ is the concentration of the redox molecules in solution. Anodic $I_{p a}$ and cathodic $I_{p c}$ peak currents were taken from the linear fit of the voltammogram where no electrochemical analyte activity was observed [16,17].

The electroactive area $(A)$ was obtained from the slope of the cathodic peak $\left(I_{p c}\right)$ versus the square root of the scan rate $(\sqrt{v})$ at a $\mathrm{K}_{4} \mathrm{Fe}(\mathrm{CN})_{6}$ concentration of $5 \mathrm{mM}$ referring to the previous mentioned expression (Eq. (2)) [16]. In addition, considering once again the Randles-Sevcik equation, the sensitivity $(S)$ and detection limit were computed since these are two important parameters in sensing applications. The sensitivity per electrode area was evaluated from the angular coefficient of the straight line obtained by plotting current density values versus the $\mathrm{K}_{4} \mathrm{Fe}(\mathrm{CN})_{6}$ concentration $[4,17]$. To evaluate this parameter, we varied the target concentration from 0 to $25 \mathrm{mM}$ by steps of $5 \mathrm{mM}$ at a scan rate of $100 \mathrm{mV} / \mathrm{s}$. The detection limit was computed as three times the signal-to-noise ratio [18] using the expression $3 \delta \mathrm{i} / \mathrm{S}$, where $\delta \mathrm{i}$ is the mean square root deviation of the voltammogram lines with no electrochemical analyte activity and performed for different target concentrations. The electron transfer rate constant $\left(k_{e t}\right)$ was computed by varying the scan rate over the range $25-150 \mathrm{mV} / \mathrm{s}$ according to the Laviron model [19].

\section{Results and discussion}

\subsection{SEM images and contact angle measurements}

Fig. 1 depicts FE-SEM images of MWCNTs directly grown on silicon wafer. Fig. 1(A) and (B) shows the aggregates of CNTs with exposed sidewalls alternating with areas of more horizontally and randomly oriented tubes. In this case, both walls and tips are in contact with the solution during electrochemical experiments. On the other hand, the layer of vertically oriented MWCNTs exposes only the edge ends to the solution (Fig. 1(C)).

Pristine MWCNTs show a hydrophobic behavior because of their size and intrinsic disorder [20]. The contact angles before and after acid treatment are summarized in Table 1 . Acid treatment causes a large improvement in HRO CNT wettability (average contact angle: $62^{\circ} \pm 5^{\circ}$ ). Conversely, VA CNTs maintain their characteristic hydrophobicity
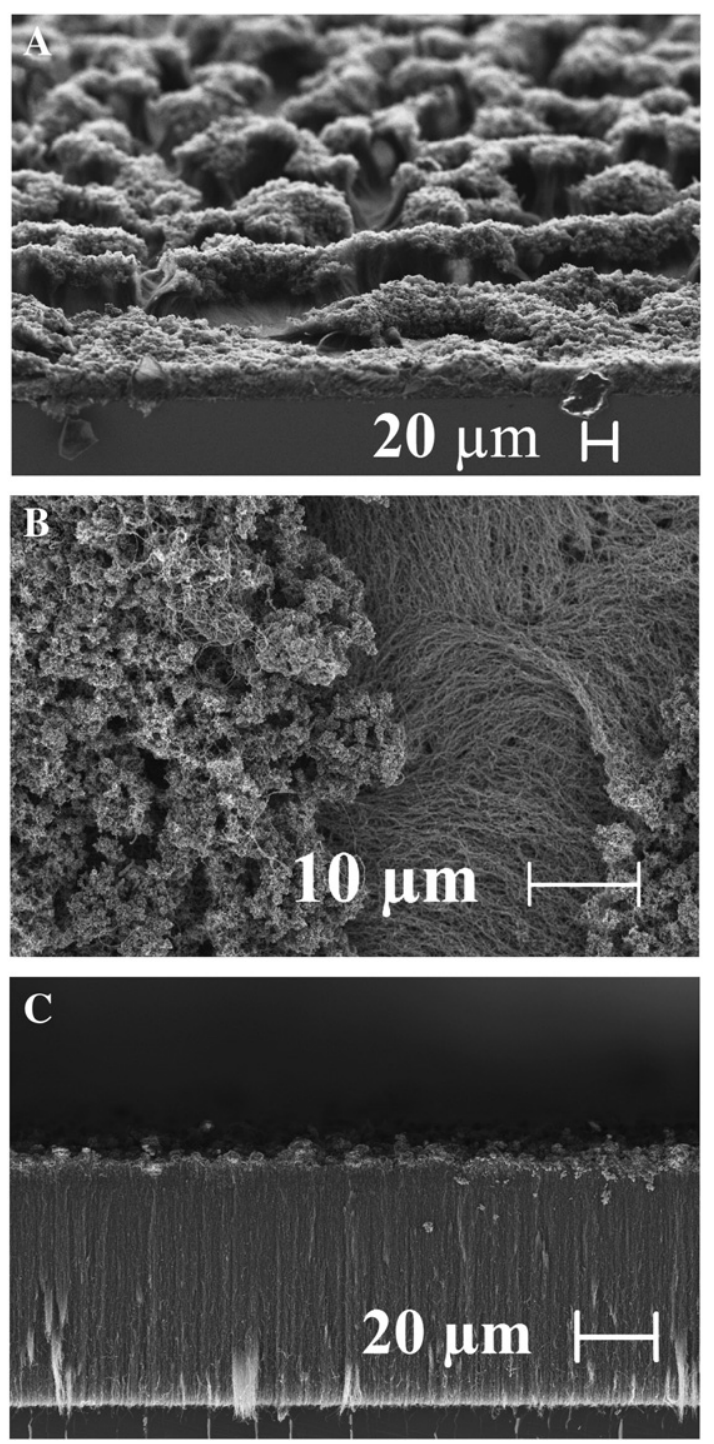

Fig. 1. FE-SEM images of HRO CNTs at lower (A) and higher (B) magnitudes and FESEM image of VA CNTs (C). 
Table 1

Contact angles before and after the treatment

\begin{tabular}{llr}
\hline & Before & After \\
\hline VA & $109^{\circ} \pm 2^{\circ}$ & $107^{\circ} \pm 4^{\circ}$ \\
HRO & $108^{\circ} \pm 6^{\circ}$ & $62^{\circ} \pm 5^{\circ}$ \\
\hline
\end{tabular}

after the treatment (average contact angle: $107^{\circ} \pm 4^{\circ}$ ). These results confirm the assumption that acid treatment improves the wettability of CNTs acting on their sidewalls [9].

\subsection{Electrochemical characterization}

In order to investigate the electrochemical properties of treated MWCNTs, the two types of CNT-based electrodes were characterized by $\mathrm{CV}$ in $\mathrm{K}_{4} \mathrm{Fe}(\mathrm{CN})_{6}$ solutions. Cyclic voltammograms obtained with different scan rates in solutions of $\mathrm{K}_{4} \mathrm{Fe}(\mathrm{CN})_{6} 5 \mathrm{mM}$ are reported in Fig. 2(A) for VA CNTs and (B) for HRO CNTs. In both cases, cathodic peak currents increase with increasing potential scan rate and are linearly related with the square root of the scan rates in the range of 25$150 \mathrm{mV} / \mathrm{s}$ (see the insets in Fig. 2) suggesting a diffusion controlled process of reactants at the electrode surface $[21,22,23]$.

Comparing the curves at $100 \mathrm{mV} / \mathrm{s}$ in the two different series of cyclic voltammograms of Fig. 2, we see that the peak current ratio is equal to unity for vertically packed CNTs $\left(I_{p a} / I_{p c} \approx 1\right)$ while HRO CNTs exhibit a $I_{p a} / I_{p c}$ equal to 1.3 . The half-wave potentials $E_{1 / 2}$ were determined from the anodic $\left(E_{p a}\right)$ and cathodic $\left(E_{p c}\right)$ peak potentials by using the expression $\left(E_{p a}+E_{p c}\right) / 2$. The peak-to-peak
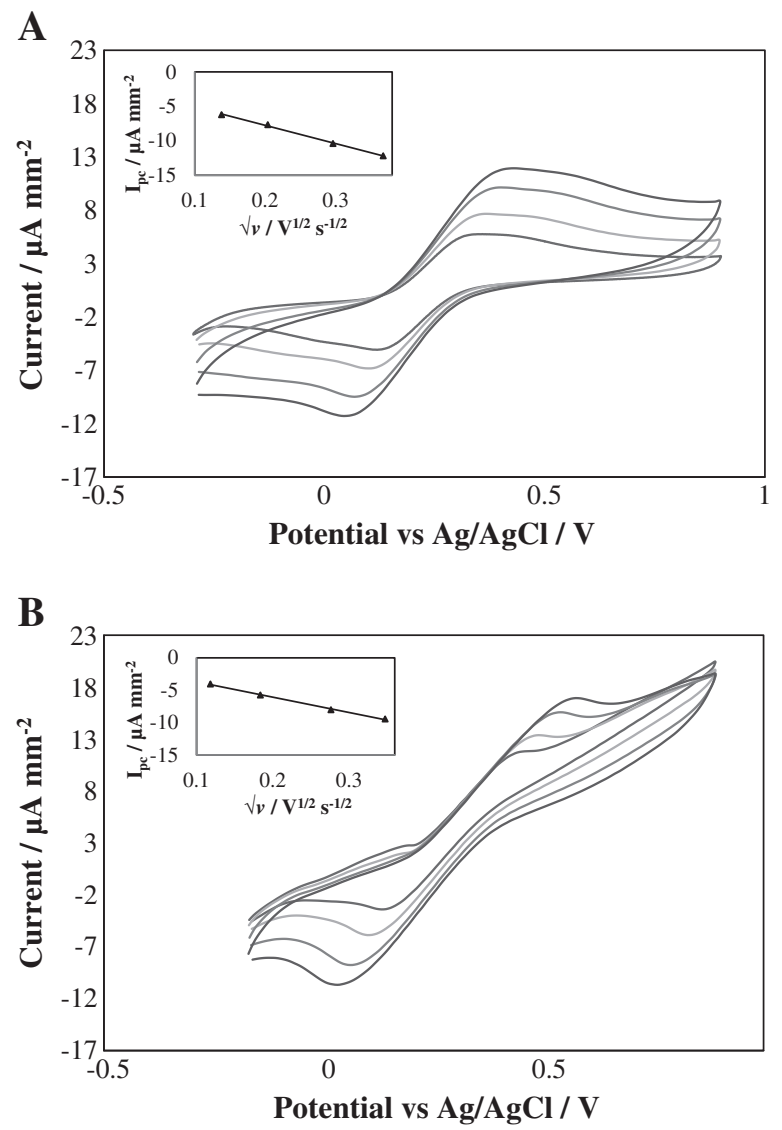

Fig. 2. Cyclic voltammograms at VA (A) and HRO (B) MWCNTs in 0.01 PSB containing $\mathrm{K}_{4} \mathrm{Fe}(\mathrm{CN})_{6} 5 \mathrm{mM}$ at scan rates of $25,50,100$ and $150 \mathrm{mV} / \mathrm{s}$. The insets show the linear relationship between the cathodic peak currents and the square root of the scan rates. separation $\left(\Delta E_{p}\right)$ is lower by $114 \mathrm{mV}$ where VA MWCNTs are used. Values of the previous mentioned electrochemical parameters for the two systems are listed in Table 2. Since the peak-to-peak separation is directly correlated to the electron transfer kinetic, these findings indicate that the most reversible and the fastest electron transfer electrochemical reaction occurs at VA CNT-based electrode. Note that the peak separation is large $(>300 \mathrm{mV})$, that means a high resistance with both the electrodes [22]. In general, low kinetics of the electron transfer process are not dependent on the analyte concentration. On the other hand, an uncompensated resistivity depends on the concentration. Registering CVs at different concentrations, we observed an increase of $\Delta E_{p}$ that, accordingly, can be attributed to the uncompensated resistance (examples in Fig. 4). Since the amount of uncompensated resistance is almost the same for the two CNTsbased electrodes, the $\Delta E_{p}$ were used to obtain an approximative value of the kinetic parameters with the aim to compare the behavior of the two electrodes. These parameters were evaluated following the Laviron model [19].

The peak potentials can be described by:

$E_{p c}=E^{0^{\prime}}-\frac{2.303 R T}{\alpha n F} \cdot \log \frac{\alpha}{m}$,

$E_{p a}=E^{0^{\prime}}+\frac{2.303 R T}{(1-\alpha) n F} \cdot \log \frac{(1-\alpha)}{m}$,

with $m=(R T / F)\left(k_{e t} / n v\right) . R$ is the gas constant, $n$ is the number of electrons involved in the redox reaction, $T$ is the absolute temperature in Kelvin, $F$ is the Faraday constant, $v$ is the scan rate, and $E^{0^{\prime}}$ is the surface standard potential. The transfer coefficient $\alpha$ can be determined according to the slopes of the regression straight lines obtained by plotting $E_{p a}$ and $E_{p c}$ vs $\log v$ (Fig. 3(A) and (B)). The electron transfer constant rate $k_{e t}$ was obtained by introducing the values $\alpha$ in the following expression

$\log k_{e t}=\alpha \log (1-\alpha)+(1-\alpha) \log \alpha-\log \frac{R T}{n v F}-\alpha(1-\alpha) \frac{n F \Delta E_{p}}{2.3 R T}$.

Based on Eq. (5) and from $\Delta E_{p}$ vs $\log v$ (Fig. 3 - insets), we obtained electron transfer rate constants of $0.18 \pm 0.02 \mathrm{~s}^{-1}$ and $0.022 \pm$ $0.001 \mathrm{~s}^{-1}$ for VA and HRO CNTs, respectively. In vertically packed CNTs, only the tips are electrochemical active sites [24]. Alternatively, both exposed tips and edge-like sidewall defects created with the acid treatment [9] contribute to the electroactivity for HRO CNTs. We suppose that the different kinetics are due to the larger density of the active sites, namely CNT tips, in VA nanotubes and also to the basal plane-like structure of the other CNT system [8].

VA CNTs also show a slightly higher electroactive area computed by using Eq. (2). Indeed, HRO CNTs have an electroactive to real area ratio of $(74 \pm 2) 10^{-4}$ and VA nanotubes of $(83 \pm 2) 10^{-4}$.

Important parameters for applications in sensors, namely sensitivity and detection limit, were also computed in reference to cathodic peaks by using voltammograms obtained for concentrations of $\mathrm{K}_{4} \mathrm{Fe}(\mathrm{CN})_{6}$ in the range $5-25 \mathrm{mM}$. For dense VA MWCNTs, the sensitivity is $71.5 \pm$ $0.3 \mu \mathrm{A} /\left(\mathrm{mM} \mathrm{cm}^{2}\right)$, slightly higher than for HRO nanotubes $(62.8 \pm$ $\left.0.3 \mu \mathrm{A} /\left(\mathrm{mM} \mathrm{cm}^{2}\right)\right)$, as in the case of electroactive to real area ratios.

Table 2

Electrochemical parameters characterizing the redox couple $\left[\mathrm{Fe}(\mathrm{CN})_{6}\right]^{-3} /\left[\mathrm{Fe}(\mathrm{CN})_{6}\right]^{-4}$ on VA and HRO CNT-based electrodes $\left(\mathrm{K}_{4} \mathrm{Fe}(\mathrm{CN})_{6}\right.$ concentration: $5 \mathrm{mM}$; scan rate: $100 \mathrm{mV} / \mathrm{s}$ ).

\begin{tabular}{|c|c|c|c|c|}
\hline & $E_{p a}$ & $E_{p c}$ & $\Delta E_{p}$ & $E_{1 / 2}$ \\
\hline & {$[\mathrm{mV}]$} & {$[\mathrm{mV}]$} & {$[\mathrm{mV}]$} & {$[\mathrm{mV}]$} \\
\hline VA & $395.04 \pm 0.01$ & $62.876 \pm 0.002$ & $332.16 \pm 0.02$ & $228.96 \pm 0.01$ \\
\hline HRO & $468.95 \pm 0.01$ & $22.696 \pm 0.001$ & $446.26 \pm 0.03$ & $245.83 \pm 0.01$ \\
\hline
\end{tabular}


A
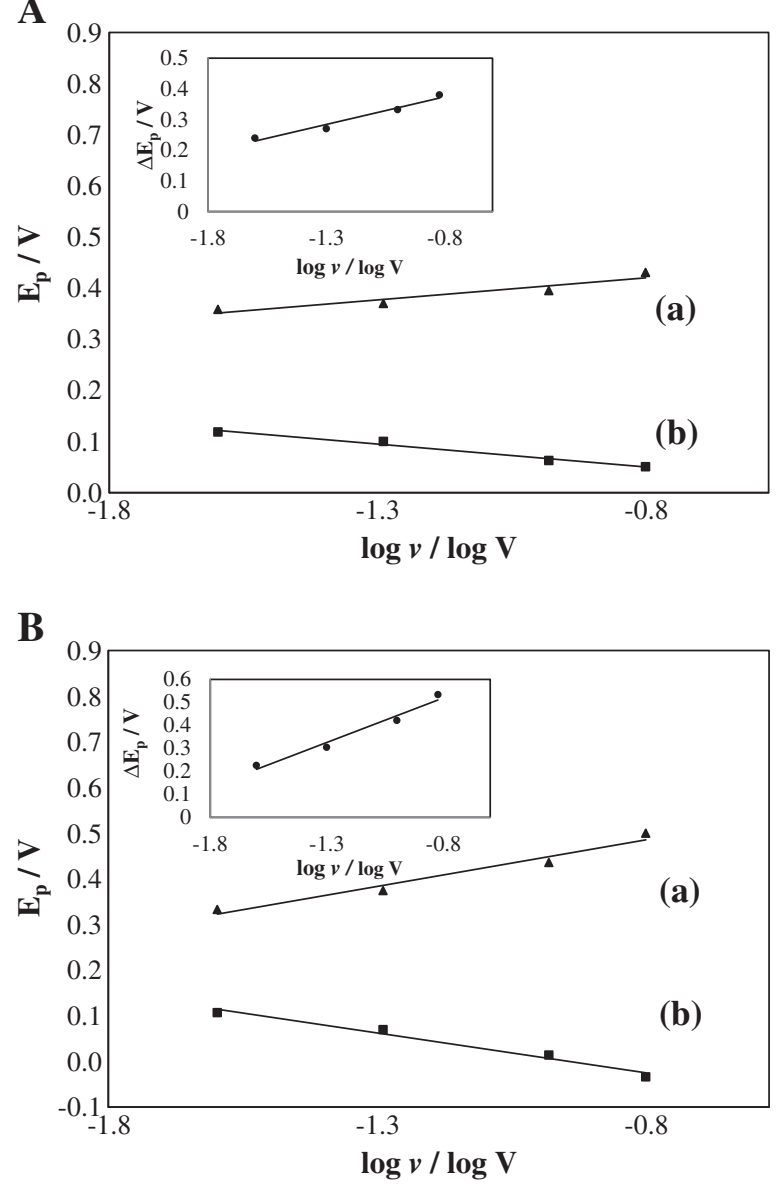

Fig. 3. Plots of $E_{p a}$ (a) and $E_{p c}$ (b) upon the logarithm of the scan rate for VA (A) and HRO (B) CNTs. Insets: plot of $\Delta E_{p}$ as a function of $\log v$.

Coherently, the lowest detection limit was obtained for the system with a vertical orientation of the nanotubes $(28.233 \pm 0.003 \mu \mathrm{M})$, while for the horizontal-CNT based electrode we obtained a detection limit of $78.56 \pm 0.03 \mu \mathrm{M}$

\section{Conclusions}

We synthesized VA MWCNTs and HRO MWCNTs onto silicon wafers using a CVD method. Contact angle data demonstrate the acid treatment effect on CNT sidewalls due to the wettability improvement only in the case of CNTs with exposed sidewalls.

The current peak ratio, peak-to-peak potential separation, and kinetic study demonstrate a slightly faster potassium ferricyanide reaction at VA CNT-based electrode. The increase in electroactive sites on HRO CNTs following the acid activation allows to obtain values of sensitivity, detection limit, and electroactive to real area ratio similar to those obtained with VA nanotubes.

Our findings demonstrate that the measured global sensitivity and detection limit of the two different surfaces are almost equal, although other authors [8] have shown kinetics greatly larger from the tips rather than from the lateral side. However, the data presented in this work provide the first experimental proof of previous theoretical simulations [10].

Future developments of this work can be the electrochemical study of other orientations of the nanotubes onto the silicon substrate as well as the same study performed with molecules well-established electrochemically such as the ascorbic acid [15] in order to gain fundamental insight about the electrosensing behavior of HRO and VA MWCNTs.
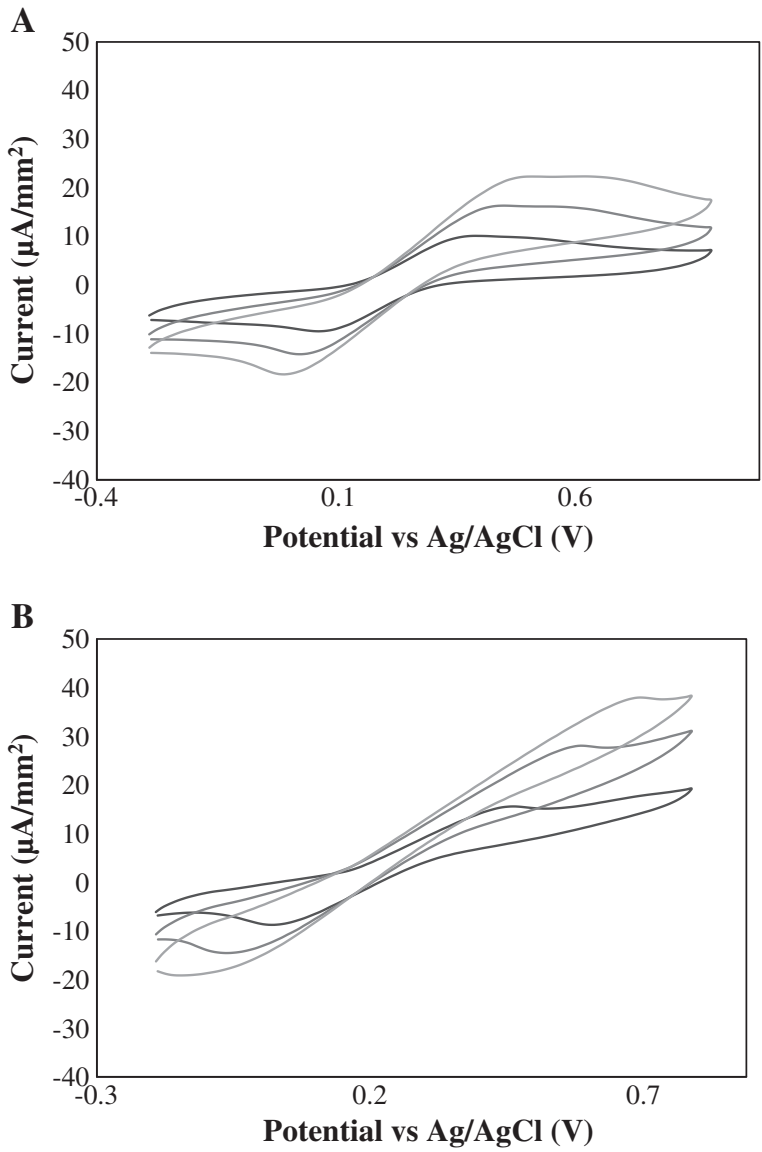

Fig. 4. Voltammograms obtained for $5,10,20 \mathrm{mM}$ of $\mathrm{K}_{4} \mathrm{Fe}(\mathrm{CN})_{6}$ concentrations at VA (A) and HRO CNTs (B).

\section{Acknowledgment}

The authors would like to thank Giuseppe Dino Albini for the useful discussion on the electrochemical properties of differently oriented carbon nanotubes and Salvatore Guastella for the acquisition of FESEM images. The research was supported by the i-IronIC project. The i-IronIC project was financed with a grant from the Swiss Nano-Tera.ch initiative and evaluated by the Swiss National Science Foundation. This work was also partially supported by LATEMAR, Center of Excellence funded by the Italian Ministry for Education, University and Research.

\section{References}

[1] R.H. Baughman, A.A. Zakhidov, W.A. de Heer, Science 297 (2002) 787.

[2] J. Liu, A. Chou, W. Rahmat, M.N. Paddon-Row, J.J. Gooding, Electroanalysis. 17 (2005) 38.

[3] F. Valentini, A. Amine, S. Orlanducci, M.L. Terranova, G. Palleschi, Anal. Chem. 75 (2003) 5413.

[4] S. Carrara, V.V. Shumyantseva, A.I. Archakov, B. Samori, Biosens. Bioelectron. 24 (2008) 148

[5] V. Bavastrello, E. Stura, S. Carrara, V. Erokhin, C. Nicolini, Sens. Actuators, B 98 (2004) 247.

[6] K. Balasubramanian, M. Burghard, Anal. Bioanal. Chem. 385 (2006) 452.

[7] M. Pumera, T. Sasaki, H. Iwai, Chem. Asian J. 3 (2008) 2046.

[8] R.R. Moore, C.E. Banks, R.G. Compton, Anal. Chem. 76 (2004) 2677

[9] F.C. Moraes, M.F. Cabral, L.H. Mascaro, S.A.S. Machado, Surf. Sci. 605 (2011) 435

[10] C. Boero, S. Carrara, G. Del Vecchio, L. Calzà, G. De Micheli, IEEE Trans. Nanobioscience 10 (2011) 59.

[11] M. Kumar, Y. Ando, Chem. Phys. Lett. 374 (2003) 521.

[12] M. Kumar, Y. Ando, J. Phys. Conf. Ser. 61 (2007) 643.

[13] S. Porro, S. Musso, M. Giorcelli, A. Chiodoni, A. Tagliaferro, Physica E 37 (2007) 16.

[14] Y.S. Chen, J.H. Huang, C.C. Chuang, Carbon 47 (2009) 3106.

[15] P. Chen, R.L. McCreery, Analytical Chemistry. 68 (1996) 3958. 
[16] A.J. Bard, L.R. Faulkner, Electrochemical Methods: Fundamentals and Applications, second ed. Wiley, New York, 2001.

[17] S. Miserendino, J. Yoo, A. Cassell, Y.-C. Tai, Nanotechnology 17 (2006) S23.

[18] J. Mocak, A.M. Bond, S. Mitchell, G. Scollary, Pure Appl. Chem. 69 (1997) 297.

[19] E. Laviron, J. Electroanal. Chem. 101 (1979) 19.

[20] K.K.S. Lau, J. Bico, Kenneth B.K. Teo, M. Chhowalla, G.A.J. Amaratunga, W.I. Milne, G.H. McKinley, K.K. Gleason, Nano Lett. 3 (2003) 1701.
[21] L. Weber, K. Kloeckner, U. Ritter, P. Scharff, Russ. J. Electrochem. 45 (2009) 1145.

[22] N. Tsierkezos, U. Ritter, J. Solid State Electrochem. 14 (2010) 1101.

[23] D. Johnson, B. Lewis, D. Elliot, J. Miners, L. Martin, Biochem. Pharmacol. 69 (2005) 1533.

[24] M. Pumera, Chem. Eur. J. 15 (2009) 4970. 